\title{
Thoracic Air Leak Syndrome: A Rare and Fatal Complication of Allogeneic Stem Cell Transplant
}

\author{
Abdullah Sakkat1*, Matthew Patel'2, Shariq Haider'1 \\ ${ }^{1}$ Department of Medicine, McMaster University, Hamilton, Canada \\ ${ }^{2}$ School of Medicine, Royal College of Surgeons in Ireland, Dublin, Ireland \\ Email: ^abdullah.sakkat@hotmail.com
}

How to cite this paper: Sakkat, A., Patel, M. and Haider, S. (2020) Thoracic Air Leak Syndrome: A Rare and Fatal Complication of Allogeneic Stem Cell Transplant. Case Reports in Clinical Medicine, 9, 81-86. https://doi.org/10.4236/crcm.2020.94013

Received: March 11, 2020

Accepted: March 30, 2020

Published: April 2, 2020

Copyright ( 2020 by author(s) and Scientific Research Publishing Inc. This work is licensed under the Creative Commons Attribution International License (CC BY 4.0).

http://creativecommons.org/licenses/by/4.0/ (c) (i) Open Access

\begin{abstract}
Thoracic air leak syndrome (TALS) is a rare complication of allogeneic stem cell transplant (Allo-SCT) and is associated with a poor prognosis. We report a case of a 67-year-old male, after 20 months of Allo-SCT for acute myeloid leukemia, who presented with fever and acute worsening dyspnea on a background of a 4-month history of slowly progressive dyspnea. He was initially diagnosed with parainfluenza pneumonia and later developed TALS as a result of bronchiolitis obliterans (BO) caused by chronic graft-versus-host disease (cGVHD). Herein, we highlight the importance of early recognition and treatment of non-infectious pulmonary complications of Allo-SCT.
\end{abstract}

\section{Keywords}

Thoracic Air Leak Syndrome, Pneumomediastinum, Bronchiolitis Obliterans, Graft Versus Host Disease, Allogeneic Stem Cell Transplant

\section{Introduction}

Allogeneic stem cell transplant (Allo-SCT) is the treatment of choice for many malignant and nonmalignant hematological disorders. Pulmonary complications of post Allo-SCT are common and are associated with a significant increase in morbidity and mortality [1] [2] [3]. Bronchiolitis obliterans (BO) is a late non-infectious pulmonary complication post Allo-SCT with a reported prevalence rate of 5.5\% in one study enrolled 1145 patients. This study also identified multiple risk factors for $\mathrm{BO}$ and found that chronic graft-versus-host disease (cGVHD) is the most important risk factor leading to a rise in the prevalence rate to $14 \%$ [4]. Yet, the etiology of BO post Allo-SCT remains unclear. 
TALS was first defined by Franquet et al. as the presence of extra-alveolar air in the thoracic cavity, which includes conditions such as spontaneous pneumothorax, pneumomediastinum, pneumopericardium, subcutaneous emphysema and interstitial emphysema [5]. The mechanism of TALS is unknown but thought to be caused by the rupture of the alveoli leading to interstitial emphysema, which leads to air travel along the bronchovascular sheath to the mediastinum [6]. The fixed airflow obstruction seen in BO could lead to distal overdistention and rupture of the alveoli, which similarly permits air entry to the bronchovascular sheath. The real incidence of TALS in patients with BO remains unclear due to the lack of reported cases. The largest study in the literature estimated an incidence rate of $0.83 \%$ [7]. Multiple risk factors have been identified for TALS post Allo-SCT. The most important risk factors are grade III-IV acute GVHD, extensive cGVHD, and pulmonary invasive fungal infection [8]. Herein, we report a case of TALS in a patient with BO and describe imaging findings, pulmonary function tests (PFTs) results and clinical course.

\section{Case Presentation}

A 67-year-old male underwent non-myeloablative Allo-SCT for the treatment of acute myeloid leukemia (see timeline, Figure 1). The conditioning regimen consisted of fludarabine and total body irradiation. Both the donor and recipient were CMV seropositive with the same blood group (O positive) and a 10/10 match. The patient had successful engraftment with no evidence of graft versus host disease (GVHD) or respiratory complications in the last clinic visit to his oncologist and pulmonologist at 16 months post-transplant.

Post-transplant screening PFTs revealed an isolated reduction in the diffusing

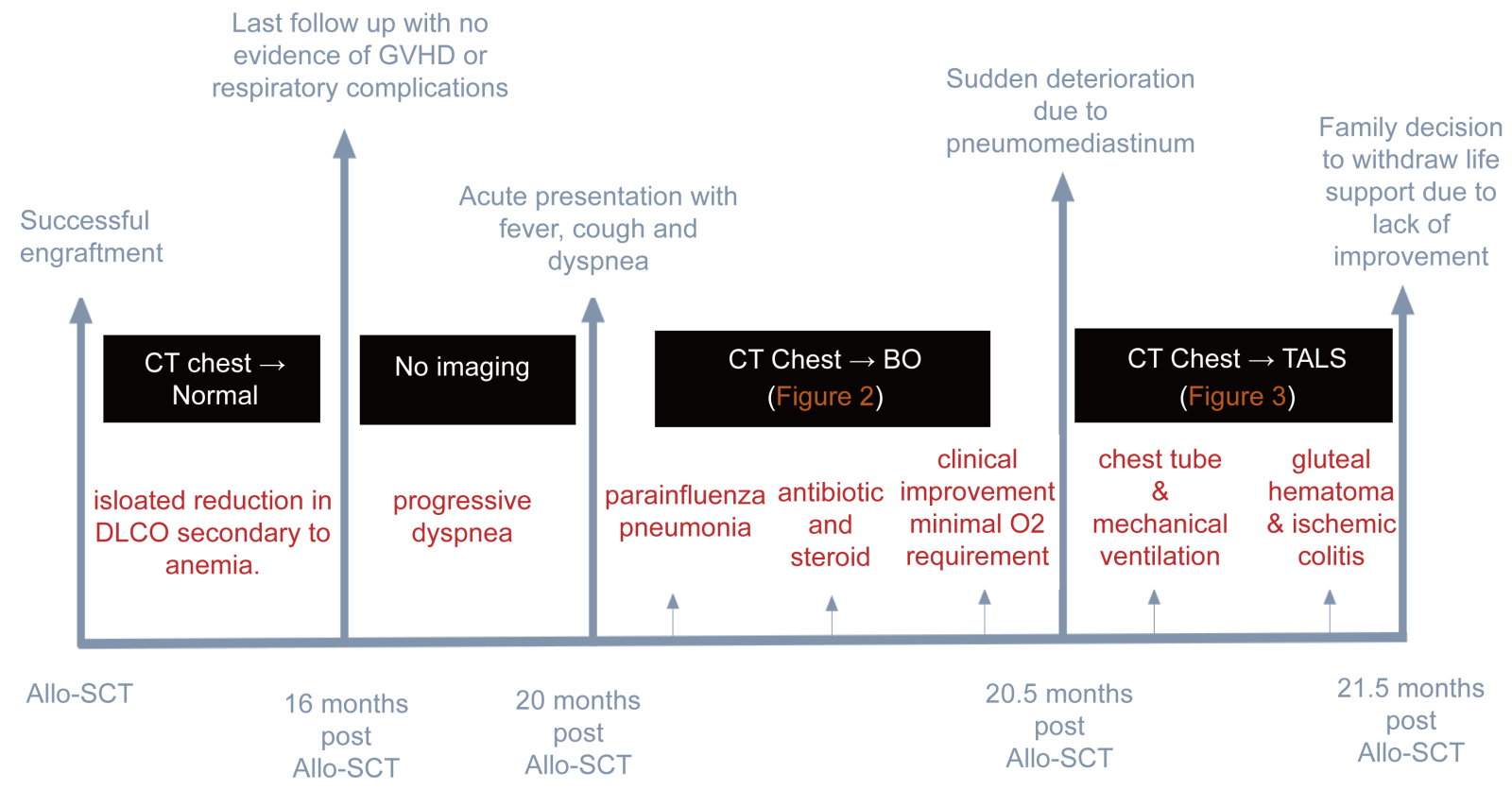

Figure 1. Timeline of important clinical information from the patient's case. 
capacity of the lungs for carbon monoxide (DLCO), which was further investigated by a computed tomography (CT) scan of the chest that was normal with no evidence of pulmonary complications. The reduction in DLCO was believed to be secondary to anemia as measurements were not corrected to hemoglobin. Serial PFTs were performed to monitor changes in DLCO over time. They demonstrated a progressive reduction in total lung capacity (TLC) and an increase in residual volume (RV) to TLC ratio with no evidence of airflow obstruction.

Twenty months post Allo-SCT, the patient presented to a community hospital with a 2-day history of fever, cough and acute worsening dyspnea on the background of slowly progressive dyspnea over the last 4 months. CXR showed right middle and lower zone opacities. The nasopharyngeal swab was positive for parainfluenza virus, and blood cultures were negative. He was admitted to the hospital and treated with azithromycin and oxygen. He rapidly deteriorated in the first 48 hours, with a significant increase in oxygen requirements. A CT of the chest (Figure 2) at this time performed and showed new findings of mosaic attenuation, bronchiectasis, airway thickening and ground-glass opacities in keeping with pneumonia and new radiographic features suggestive of BO.

The patient was then transferred to our institution for further management. He was admitted to the ICU and treated with systemic corticosteroids and piperacillin/tazobactam. The patient's condition improved and stabilized over time with minimal oxygen requirement. On day 14 post admission, the patient had a sudden respiratory deterioration with an increase in oxygen requirement,

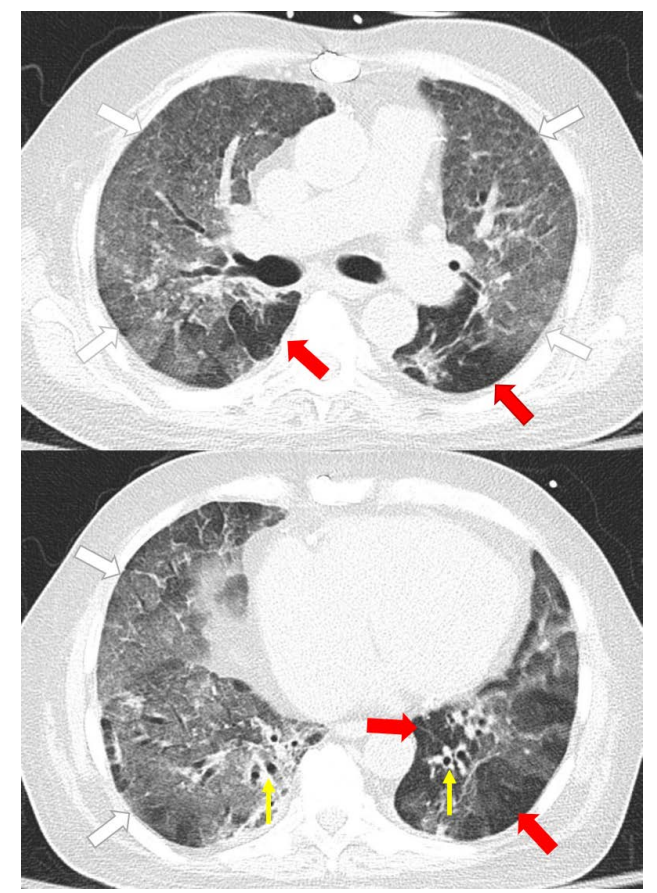

Figure 2. High-resolution CT scan of the chest showing features of BO: mosaic attenuation (red arrows), bronchiectasis and airway thickening (yellow arrows) diffuse ground-glass opacity (white arrows). 
which led to urgent intubation. A chest X-ray at that moment demonstrated radiographic findings suspicious of pneumomediastinum. A subsequent Chest CT scan (Figure 3) confirmed the presence of pneumomediastinum with no definitive source of the air leak. Oral contrast study showed no evidence of esophageal perforation. Bilateral chest tubes were inserted, and mechanical ventilation was continued using lung-protective ventilation strategy. The patient continued to have a persistent air leak, and unfortunately, his ICU course was further complicated by a large gluteal hematoma and ischemic colitis. Given the complicated course and no signs of improvement after four weeks of treatment, his family decided to withdraw life support measures.

\section{Discussion}

Chronic GVHD is a common serious complication post Allo-SCT occurring in $60 \%-80 \%$ of patients [9] [10]. It is a multisystem disease with pulmonary involvement occurring approximately in half of the patients [11]. It may manifest as $\mathrm{BO}$ with a fixed obstructive defect and/or bronchiolitis obliterans organizing pneumonia (BOOP) with a mixed obstructive and restrictive defect.

$\mathrm{BO}$ onset is usually insidious, with a non-productive cough and dyspnea. It is characterized clinically by fixed airflow obstruction and gas trapping, histologically by concentric bronchiolar lumen narrowing by submucosal fibrous tissue and radiographically by mosaic attenuation, bronchiectasis, and bronchial wall thickening [5] [12] [13]. The gold standard diagnostic modality of BO is lung biopsy. Because of the invasive nature and complications related to lung biopsy, $\mathrm{BO}$ is alternatively diagnosed by a combination of PFT findings, radiographic

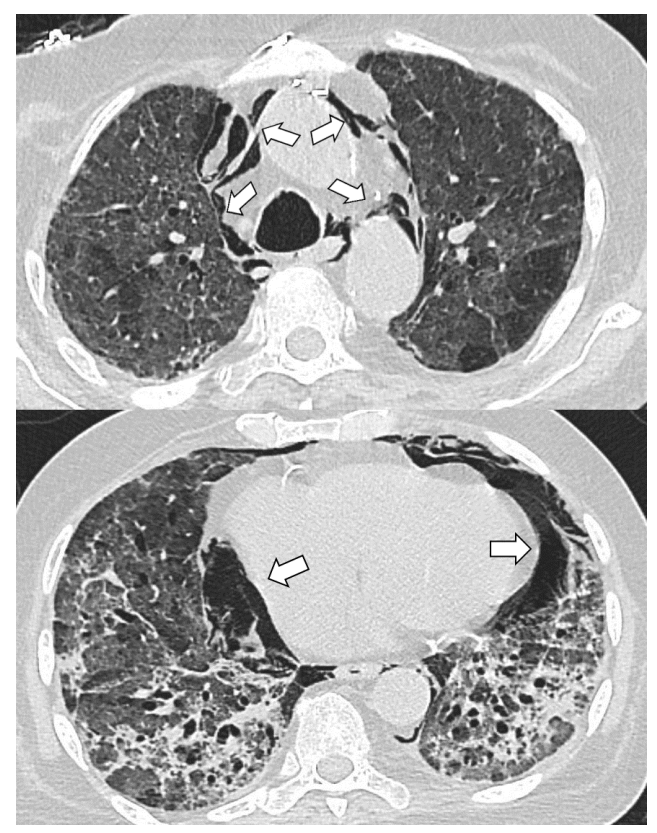

Figure 3. CT scan of the chest showing interval development of pneumomediastinum extending from the thoracic inlet to the diaphragm (white arrows). 
features and at least one other distinctive manifestation in a separate organ [14]. One of the limitations in our case is the lack of a confirmatory histological diagnosis of BO and airflow obstruction on PFT. Our patient was too ill to complete these procedures, and there was no follow up for the four months preceding the presentation, which might have led to a delay in diagnosis. The initial coexistence of parainfluenza pneumonia could be a red herring. Although it could have complicated the case but is likely unrelated to TALS as the radiographic features were highly suggestive of $\mathrm{BO}$, and the clinical course was not fully explained by parainfluenza pneumonia alone.

In our case, we ruled out other potential causes of TALS, such as aspergillus lung infection and pneumocystis pneumonia. Treatment of TALS in patients with $\mathrm{BO}$ is primarily supportive with the insertion of a chest tube and supplemental oxygen with or without ventilatory support. Once TALS developed, it is unlikely that the treatment of $\mathrm{BO}$ will be effective due to the irreversible damage to the bronchiole, and lung transplant will possibly be the only curative treatment if the patient survived the acute period. The development of TALS in patients post Allo-SCT is associated with a poor prognosis. Previous reports in the literature describe a high mortality rate ranging between $66.7 \%-100 \%$ [7] [15]. Although our case clinical course was complicated by large gluteal hematoma and ischemic colitis, TALS with persistent air leak likely contributed to overall poor patient outcomes.

\section{Conclusion}

This case highlights the importance of early recognition and treatment of noninfectious pulmonary complications of Allo-SCT. Close follow up and serial assessment with physical examination, PFTs and imaging, if indicated, is warranted in this high-risk population. The early recognition and treatment of BO may reduce the risk of developing TALS, which associated with high mortality.

\section{Conflicts of Interest}

The authors declare no conflicts of interest regarding the publication of this paper.

\section{References}

[1] Forman, S.J., Negrin, R.S., Antin, J.H. and Appelbaum, F.R. (2016) Thomas' Hematopoietic Cell Transplantation. 5th Edition, Wiley, Hoboken, NJ. https://doi.org/10.1002/9781118416426

[2] Battiwalla, M., Hashmi, S., Majhail, N., et al. (2017) National Institutes of Health Hematopoietic Cell Transplantation Late Effects Initiative: Developing Recommendations to Improve Survivorship and Long-Term Outcomes. Biology of Blood and Marrow Transplantation, 23, 6-9. https://doi.org/10.1016/j.bbmt.2016.10.020

[3] Soubani, A.O. and Uberti, J.P. (2007) Bronchiolitis Obliterans Following Haematopoietic Stem Cell Transplantation. European Respiratory Journal, 29, 1007-1019. https://doi.org/10.1183/09031936.00052806 
[4] Au, B.K., Au, M.A. and Chien, J.W. (2011) Bronchiolitis Obliterans Syndrome Epidemiology after Allogeneic Hematopoietic Cell Transplantation. Biology of Blood and Marrow Transplantation, 17, 1072-1078.

https://doi.org/10.1016/j.bbmt.2010.11.018

[5] Franquet, T., Rodríguez, S., Hernández, J.M., Martino, R., Giménez, A., Hidalgo, A., et al. (2007) Air-Leak Syndromes in Hematopoietic Stem Cell Transplant Recipients with Chronic GVHD: High Resolution CT Findings. Journal of Thoracic Imaging, 22, 335-340. https://doi.org/10.1097/RTI.0b013e3180cab6cf

[6] Wintermark, M. and Schnyder, P. (2001) The Macklin Effect: A Frequent Etiology for Pneumomediastinum in Severe Blunt Chest Trauma. Chest, 120, 543-547.

https://doi.org/10.1378/chest.120.2.543

[7] Moon, M.H., Sa, Y.J., Cho, K.D., Jo, K.H., Lee, S.H. and Sim, S.B. (2010) Thoracic Air-Leak Syndromes in Hematopoietic Stem Cell Transplant Recipients with Graft-versus-Host Disease: A Possible Sign for Poor Response to Treatment and Poor Prognosis. Journal of Korean Medical Science, 25, 658-662.

https://doi.org/10.3346/jkms.2010.25.5.658

[8] Chien, S.-H., et al. (2016) Spontaneous Post-Transplant Air-Leak Syndrome in Adult Patients Receiving Allogeneic Hematopoietic Stem Cell Transplantation. Blood, 128, 5780-5780. https://doi.org/10.1182/blood.V128.22.5780.5780

[9] Nash, R.A., Antin, J.H., Karanes, C., et al. (2000) Phase 3 Study Comparing Methotrexate and Tacrolimus with Methotrexate and Cyclosporine for Prophylaxis of Acute Graft-versus-Host Disease after Marrow Transplantation from Unrelated Donors. Blood, 96, 2062-2068.

[10] Ratanatharathorn, V., Nash, R.A., Przepiorka, D., et al. (1998) Phase III Study Comparing Methotrexate and Tacrolimus (Prograf, FK506) with Methotrexate and Cyclosporine for Graft-versus-Host Disease Prophylaxis after HLA-Identical Sibling Bone Marrow Transplantation. Blood, 92, 2303-2314.

[11] Jacobsohn, D.A., Kurland, B.F., Pidala, J., Inamoto, Y., Chai, X., Palmer, J.M., Arai, S., Arora, M., Jagasia, M., Cutler, C., Weisdorf, D., Martin, P.J., Pavletic, S.Z., Vogelsang, G., Lee, S.J. and Flowers, M.E. (2012) Correlation between NIH Composite Skin Score, Patient-Reported Skin Score, and Outcome: Results from the Chronic GVHD Consortium. Blood, 120, 2545-2552. https://doi.org/10.1182/blood-2012-04-424135

[12] Allen, T.C. (2010) Pathology of Small Airways Disease. Archives of Pathology \& Laboratory Medicine, 134, 702-718.

[13] Pipavath, S.J., Lynch, D.A., Cool, C., Brown, K.K. and Newell, J.D. (2005) Radiologic and Pathologic Features of Bronchiolitis. American Journal of Roentgenology, 185, 354-363. https://doi.org/10.2214/ajr.185.2.01850354

[14] Filipovich, A.H., Weisdorf, D., Pavletic, S., Socie, G., Wingard, J.R., Lee, S.J., Martin, P., Chien, J., Przepiorka, D., Couriel, D., Cowen, E.W., Dinndorf, P., Farrell, A., Hartzman, R., Henslee-Downey, J., Jacobsohn, D., McDonald, G., Mittleman, B., Rizzo, J.D., Robinson, M., Schubert, M., Schultz, K., Shulman, H., Turner, M., Vogelsang, G. and Flowers, M.E. (2005) National Institutes of Health Consensus Development Project on Criteria for Clinical Trials in Chronic Graft-versus-Host Disease: I. Diagnosis and Staging Working Group Report. Biology of Blood and Marrow Transplantation, 11, 945-956. https://doi.org/10.1016/j.bbmt.2005.09.004

[15] Vogel, M., Brodoefel, H., Bethge, W., Faul, C., Hartmann, J., Schimmel, H., et al. (2006) Spontaneous Thoracic Air-Leakage Syndrome in Patients Following Allogeneic Hematopoietic Stem Cell Transplantation: Causes, CT-Follow up and Patient Outcome. European Journal of Radiology, 60, 392-397.

https://doi.org/10.1016/j.ejrad.2006.07.013 